\title{
New Antibiotics: Where Are They?
}

\author{
Hua Deng* and Hongtao Yu \\ Department of Chemistry, Morgan State University, Maryland
}

Received: 眥: October 04, 2018; Published: 跳: October 17, 2018

*Corresponding author: Hua Deng, Department of Chemistry, Morgan State University, Baltimore, Maryland

\begin{abstract}
Multidrug resistant bacteria are marching and winning. The entire human race is at risk and new, effective antibiotics are needed for survival. Several notable routines to develop novel antibiotics are briefly compared here and possible new strategies are proposed. Key words include but not limited to nanotechnology, metal nanoparticles, natural product, medicinal plants, folk medicine, ethnopharmacology, computer-based screening, synergistic antibacterial activity, genomics, peptides, inhibitor, and structure-based virtual screening.
\end{abstract}

\section{Introduction}

\section{Urgent Need of New Antibiotics}

Threat from drug-resistant bacteria increases steadily and globally. As reported in 2016, over 700,000 people die, and millions suffer from drug-resistant pathogens each year. It is expected that 10 million people could be killed a year by 2050, based on the current rate of antibiotic approvals [1]. There are two main causes for the lack of effective, new antibiotics. First, there has been no new antibiotics developed since 1980s. Most pharmaceutical companies dropped their program for antibiotic development after the peak of antibiotic discovery going back to 1940s [2]. The other is the various forms of abuse of antibiotics. As a result, these bugs are all coming back now. WHO (World Health Organization) has launched a series of reports since 2004 on antimicrobial resistance $[3,4]$ and published a global priority list of antibiotic-resistant bacteria in 2017 [5]. Three bacteria strains ranked "critical" and six ranked "high" priorities. Two of them were even resistant to the 3rd generation antibiotics cephalosporin. These alarming situations warrant the search of new antibiotics. Various governments have put much effort in finance and legislation and even provide the industries with incentives. Below are some of the strategies for the development of new antibiotics.

\section{Modification to Current Antibiotics}

Antibiotics are categorized into four, and to some five, generations and with different types/classes. One method to develop new antibiotics is to change some functional groups or a small part of the molecule of the current antibiotics, namely analogues or derivatives. This adds new members to a specific class, but normally does not alter antibacterial mechanism. Thus, its contribution to overcome resistance is very limited although it counts majority of newly developed antibiotics. In 2010, only 2 out of 27 compounds that are in clinical trials are of new classes [6].

\section{Nanotechnology}

Nanotechnology is a powerful tool either as a vehicle to deliver antibiotics to a specific target or as a nanomaterials-based broadspectrum antimicrobial. As a drug delivery vehicle, dendrimers, polymer nanoparticles, natural product nanoparticles and many other engineered nanostructure are of interest to be fabricated as antibiotics $[7,8]$. Most well studied nanomaterials as antimicrobial agents are mainly metal and metal oxide nanoparticles. Two promising representatives are silver and titanium dioxide nanoparticles. Nano silver is commercially manufactured and widely used as antimicrobial coating, additive materials (like in packaging) and even in chicken feed, but not directly as a "medication" yet. The drawback is the unclear mechanism(s) and uncertain toxicity. Released silver ions are considered as an important pathway but they are also highly toxic to human cells [9]. It is possible that many pathways work additively and/or synergistically, making silver nanoparticles a strong and broadspectrum antibiotic [10]. It could be a new and revolutionary class of antibiotics if the mechanism(s) of toxicity is clarified and toxic properties are well-controlled. Titanium dioxide nanoparticles are widely used in food industry and are known to be nontoxic. But they are only antibacterial under light excitation (mainly UV range) which greatly limits their application [11]. The possible solution is to alter their energy level which currently is hard to achieve technically. Overall, nanotechnology and nanomaterial have unlimited potential to develop new antibiotics. But our current 
knowledge and technology is only a tip of the iceberg. Neither the government agencies nor the public would like to take the risk to market or use engineered nanomaterials as antibiotics.

\section{Medicinal Plants}

To find new antibiotics, a key step is to locate a reliable resource. Medicinal plants with no doubt are on top of the list. They started to be used since the ancient times. Human has accumulated a great deal of knowledge and experience throughout many generations, [12] and this is termed folk medicine. These medicines vary greatly on the geographical and biological composition of the plants and they have been gradually accepted and documented as ethnopharmacology. It has become a very important and integral part of pharmaceuticals, especially in Asia, Africa and South America. Chemists began to extract and isolate effective species to develop new medicines centuries ago. A standard protocol include extraction, isolation, examination of antibacterial activity, purification and determination of structure. Research groups all over the world are employing this routine to find new antibiotics. Cost is high. Procedure is very time-consuming and laborious. Unfortunately, most of the purified compounds have lower antibacterial activity compared to that of the extracts. Even so some make to clinical trials, none of the marketed antibiotics are directly isolated from medicinal plants.

\section{Microorganisms}

Microorganisms are another potential resource for antibiotics. Discovery of penicillin is the best-known example. Another example is photoradars luminesces, known as "beaming bacteria". They can produce and release antibiotics, lethal to other bacteria to protect their territory and food source. Historically, they helped the recovery of injured soldiers in the battlefield during the Civil War in 1862, by colonizing their wounds and protecting them from infection by other deadly bacteria. There they gained the nickname "Angel's Glow". Bacteria are a set of complicated creatures that follow rules set by nature and could provide beneficial guidance for the development of new antibiotics. Knowledge of their life and behavior can provide new strategy and idea for new antibiotics (this is also related to genomics and metabolism below).

The list of microorganisms is not complete without covering bacteriophage [13]. It is a type of virus that infects and replicates within bacteria and archaea by injecting of their genome into the cytoplasm of the host. There are more than 1031 bacteriophages on earth and they can be found wherever the bacteria exist. Some of them are broad-spectrum while others are specific to certain bacterial strains. They have been used as alternative antibiotics in Russian and parts of Europe for over 90 years, but not in the United States neither in most of the other countries. One reason they are not generally used is due to the discovery and wide usage of antibiotics, making bacteriophage less necessary. Another reason is poor understanding of bacteriophages and the lack of control at nanometer size level, as that of nanotechnology. But they are the natural enemies of the bacteria and they may overcome drugresistance.

\section{Genomics and Metabolism Route}

Fast development of biological technologies provides novel ideas for the development of new antibiotics, including advances in genomics research enabling the creation of non-culturable bacteria or inhibition of a specific metabolism process, etc. Genomes of bacteria are normally short comparing with that of a mammal and are relatively easy to be sequenced. The complete sequencing of the genomes guides us to explore bacteria and their resistance to antibiotics, providing fundamental information for antibiotics design. Non-culturable bacteria refer to "Viable but non-culturable bacteria" that are alive but in a state of very low metabolic activity and they do not divide. With recombinant DNA technology, it is possible to clone large fragments of the non-culturable bacterial genome and express them, [14] genetically suppressing pathogenic bacteria.

Better understanding of the bacterial cellular activity and life cycle can help designing antibiotics targeting a specific cellular component or a metabolic process, for instance, ribosome, protein synthesis, cellular respiration, cell-division, etc., [15-17]. Structural information of functional biomolecules (especially proteins or enzymes) can also provide possible route to design new antibiotics, like the active region of human defensin 5 [18]. The Nobel Prize in medicine 2018 was awarded to Drs. James P. Allison and Tasuku Honjo "for their discovery of cancer therapy by inhibition of negative immune regulation." This is a sign and example that inhibitor could be the next class of antibiotics.

\section{Computer Assisted Approaches}

Computational tools are making chemical structure screening much easier and more efficient, known as computer-based screening approach or structure-based virtual screening. This is normally combined with previous mentioned metabolism route or is based on a specific type of inhibitor (i.e. small molecule inhibitor) [19] protein and other biomolecules [20]. Besides traditional computational software, new approaches and usage of big data (i.e. peptide libraries) [21] could be a game changer and open a new page for antibiotics discovery.

\section{The Future of New Antibiotics}

No New Class of Antibiotics will be put into the Market Soon: Above lists some popular and promising routes for new antibiotics. It is always easier said than done. Discovery of potential antibiotics is a tough march. So is clinical trial and marketing process. It takes 10 - 20 years and costs at least $\$ 1$ billion to put a new antibiotic into market. As of September 2017, approximately 48 new antibiotics are in phase I (enter human testing) clinic trials [22] Only 8 of them show potential to be further tested. However, this does not bring much hope for a new antibiotic class to be marketed soon.

Abuse of Antibiotics Needs to be Stopped: To fight bacterial drug-resistance is not responsibility solely for either government agency or drug manufacturer. Instead, public should be more involved and make appropriate usage of antibiotics. This would slow down drug resistance, thus buy scientists and drug industry more time to develop new antibiotics. 
Find Clues in Nature: Mother Nature always has solutions and answers for all the problems. New class of antibiotics may be compound(s) released by some "good" bacteria in our gut, or peptide(s) from our immune defense system. It could also lie in a specific plant or natural product, like garlic, neem tree, oregano oil or even honey. Normally, scientists aim to isolate and purify a specific and effective structure from extracts. How about several species that exhibit a synergistic antibacterial effect? Given that team work is more productive than individual and is encouraged in human society, it is possible that two or more antibiotics with low antimicrobial efficiency could have synergistic antibacterial effect and overcome drug resistance. Yet, there is no report of any antibiotics based on synergistic activity of two or more active compounds. New strategies are needed to develop new antibiotics and it is an interdisciplinary task.

\section{References}

1. (2018) Wanted: a reward for antibiotic development. Nat Biotechnol 36: 555.

2. Hu C (2018) Pharmaceutical companies are backing away from a growing threat that could kill 10 million people a year by 2050 .

3. (2014) Antimicrobial resistance: global report on surveillance. World Health Organization.

4. (2000-2010) Global report on antimalarial drug efficacy and drug resistance: World Health Organization.

5. (2017) Global priority list of antibiotic-resistant bacteria to guide research discovery and development of new antibiotics. Geneva: World Health Organization.

6. Coates AR, Halls G, Hu Y (2011) Novel classes of antibiotics or more of the same. Br J Pharmacol 163(1): 184-194.

7. Hughes GA (2017) Nanomedicine in Cancer. Pan Stanford p. 47-72.

8. Du J, Du X, Mao C, Wang J (2011) Tailor-made dual pH-sensitive polymerdoxorubicin nanoparticles for efficient anticancer drug delivery. J Am Chem Soc 133(44): 17560-17563.

9. Deng H, Zhang Y, Yu H (2018) Nanoparticles considered as mixtures for toxicological research. J Environ Sci Health C 36(1): 1-20.

10. Deng H, Mc Shan, Zhang Y, Sinha S, Arslan Z, et al. (2016) Mechanistic study of the synergistic antibacterial activity of combined silver

\section{ISSN: 2574-1241}

DOI: 10.26717/BJSTR.2018.10.001909

Hua Deng. Biomed J Sci \& Tech Res

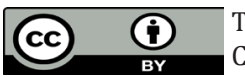

This work is licensed under Creative Commons Attribution 4.0 License

Submission Link: https://biomedres.us/submit-manuscript.php nanoparticles and common antibiotics. Environ Sci Technol 50(16): 8840-8848.

11. Brunet LN, Lyon D Y, Hotze E M, Alvarez PJ, Wiesner MR, (2009) Comparative photoactivity and antibacterial properties of $\mathrm{C} 60$ fullerenes and titanium dioxide nanoparticles. Environ Sci Technol 43(12): 43554360.

12. Petrovska B (2012) Historical review of medicinal plants usage. Pharmacogn Rev 6(11): 1.

13. Kutateladze M, Adamia R (2010) Bacteriophages as potential new therapeutics to replace or supplement antibiotics. Trends Biotechnol 28(12): 591-595.

14. Ramamurthy T, Ghosh A, Pazhani GP, Shinoda S (2014) Current perspectives on viable but non-culturable (VBNC) pathogenic bacteria. Front Public Health 2: 103.

15. Roy RN, Lomakin IB, Gagnon MG, Steitz TA (2015) The mechanism of inhibition of protein synthesis by the proline-rich peptide on cocin. Nat Struct Mol Biol 22: 466.

16. Wilson DN (2014) Ribosome-targeting antibiotics and mechanisms of bacterial resistance. Nat Rev Microbiol 12(1): 35.

17. Lobritz MA, Belenky P, Porter CB, Gutierrez A, Yang JH (2015) Antibiotic efficacy is linked to bacterial cellular respiration. Proc Natl AcadSci 112(17): 8173-8180.

18. Wang C, Shen M, Gohain N, Tolbert WD (2015) Design of a potent antibiotic peptide based on the active region of human defensin 5. J Med Chem 58(7): 3083-3093.

19. De Wealthy's E, Segers K, Sardis MF, Anne J, Nicolaes GA et al. (2015) Identification of small-molecule inhibitors against Sec A by structurebased virtual ligand screening. J Antibiot 68(11): 666.

20. Spyrakis F, Celenza G, Marcoccia F, Santucci M, Cross S (2017) Structurebased virtual screening for the discovery of novel inhibitors of New Delhi Metallo- $\beta$-lactamase-1. ACS Med Chem Lett 9(1): 45-50.

21. Tucker AT, Leonard SP, DuBois CD, Knauf GA, Cunningham AL (2018) Discovery of next-generation antimicrobials through bacterial selfscreening of surface-displayed peptide libraries.

22. (2018) Antibiotics currently in global clinical development. The PEW Charitable Trusts.

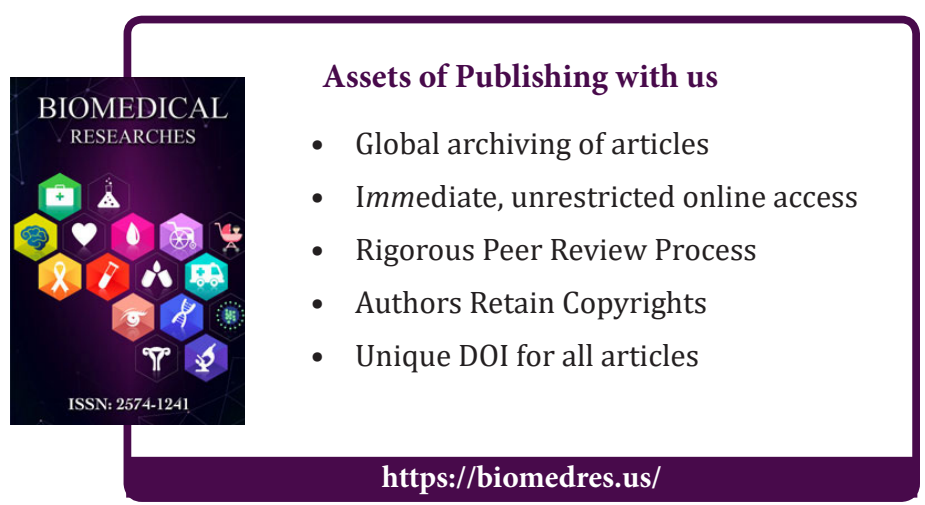

Cite this article: Hua Deng, Hongtao Yu. New Antibiotics: Where Are They?. Biomed J Sci\&Tech Res 10(1)-2018. BJSTR. MS.ID.001909. 